\title{
Salud mental en emergencias médicas: un llamado a la investigación
}

\author{
Mental health in Emergency Medicine: a call for research
}

\author{
Edgar Landa-Ramírez ${ }^{1,2 *}$ \\ ${ }^{1}$ Programa de Psicología de Urgencias, Hospital General Dr. Manuel Gea González; ${ }^{2}$ Facultad de Psicología, Universidad Nacional Autónoma de \\ México. Ciudad de México, México
}

Un estudio realizado entre 2009 y 2015, a nivel internacional, indicó que la proporción de problemas de salud mental atendidos en los Departamentos de Emergencias Médicas (DEM) aumentó entre un 40 y $60 \%$ en población adulta y pediátrica en dicho periodo ${ }^{1}$. Aunado a lo anterior, se ha identificado a los problemas de salud mental como la segunda causa más común por la cual los pacientes visitan los DEM². En el caso de América Latina, se ha reportado que hasta un 35\% de los pacientes atendidos dentro de los DEM podrían presentar algún problema de esta naturaleza; lo cual hace más probable que dichos pacientes presenten algún diagnóstico físico u otros diagnósticos de salud mental ${ }^{3,4}$.

Un trabajo realizado durante la pandemia de COVID-19 reportó un incremento importante en la atención de problemas de salud mental, intentos suicidas y consumo de sustancias entre marzo y octubre de 2020 dentro de los DEM ${ }^{5}$. La atención de estos problemas de salud mental en emergencias trae consigo una gran cantidad de consecuencias para el sistema y personal de salud; por ejemplo: saturación del servicio, sobrecarga de trabajo, elevado costo en la atención de los pacientes y mayor cantidad de horas invertidas para la atención o canalización de la necesidad ${ }^{1,6}$.
A partir de la información previamente presentada es factible colegir que a nivel mundial y nacional existe una gran cantidad de necesidad de atención a la salud mental de los pacientes que visitan los DEM; de igual forma, con base en la experiencia clínica y empírica se puede decir que existe también una gran necesidad de atención a la salud mental de los familiares y del personal de salud de las emergencias médicas ${ }^{7,8}$. Desafortunadamente, la magnitud de las necesidades reportadas contrasta con los limitados esfuerzos realizados en la región para poder abordar estas problemáticas.

Debido a la situación previa, la Revista de Educación e Investigación en Emergencias ha decidido promover una convocatoria abierta para recibir artículos científicos enfocados (pero no limitados) a la evaluación, tratamiento e identificación de factores de protección o riesgo, en la temática de salud mental de pacientes, familiares y personal de salud en la medicina de emergencias hospitalaria y prehospitalaria. Es así que invitamos al personal de emergencias, psiquiatría, psicología, enfermería, trabajo social (y demás profesiones interesadas en la salud mental) a compartir los datos de sus investigaciones originales, revisiones o casos clínicos en esta plataforma. Con estas acciones, estamos seguros de que coadyuvaremos en la creación de espacios que

\footnotetext{
Correspondencia:

*Edgar Landa-Ramírez

E-mail: edgar_landa_ramirez@yahoo.com.mx

Disponible en internet: 30-07-2021

Rev Educ Investig Emer. 2021;3(2):43-44 www.medicinadeemergencias.com 2604-6520 @ 2021 Sociedad Mexicana de Medicina de Emergencias, AC. Publicado por Permanyer México SA de CV. Este es un artículo open access bajo la licencia CC BY-NC-ND (http://creativecommons.org/licenses/by-nc-nd/4.0/).
} 
Rev Educ Investig Emer. 2021;3(2)

permitan el intercambio de conocimiento basado en evidencia, que tan imperantemente se necesita en el área.

\section{Bibliografía}

1. Santillanes G, Axeen S, Lam CN, Menchine M. National trends in mental health-related emergency department visits by children and adults, 20092015. Am J Emerg Med. 2020;38(12):2536-44.

2. Hooker EA, Mallow PJ, Oglesby MM. Characteristics and trends of Emergency Department visits in the United States (2010-2014). J Emerg Med. 2019;56(3):344-51.

3. Castilla-Puentes RC, Secin R, Grau A, Galeno R, Feijo de Mello M, Pena N, et al. A multicenter study of major depressive disorder among Emergency Department patients in Latin-American countries. Depress Anxiety. 2008;25(12):E199-204.
4. Castilla-Puentes R, Secin R, Grau A, Galeno R, De Mello MF, Castilla-Puentes $S$, et al. A multicenter study of bipolar disorder among Emergency Department patients in Latin-American countries. Int J Psychiatry Med. 2011;42(1):49-67.

5. Holland KM, Jones C, Vivolo-Kantor AM, Idaikkadar N, Zwald M, Hoots B, et al. Trends in US Emergency Department visits for mental health, overdose, and violence outcomes before and during the COVID-19 pandemic. JAMA Psychiatry. 2021;78(4):372-9.

6. Baia Medeiros DT, Hahn-Goldberg S, O'Connor E, Aleman DM. Analysis of emergency department length of stay for mental health visits: A case study of a Canadian academic hospital. CJEM. 2019;21(3):374-83.

7. Landa-Ramírez E, Domínguez-Vieyra NA, Hernández-Nuñez ME, Díaz-Vásquez LP, Santana-García IA. Psychological support during COVID-19 death notifications: Clinical experiences from a hospital in Mexico. Psychol Trauma. 2020;12(5):518-20.

8. Song X, Fu W, Liu X, Luo Z, Wang R, Zhou N, et al. Mental health status of medical staff in emergency departments during the Coronavirus disease 2019 epidemic in China. Brain Behav Immun. 2020;88:60-5. 